Review Article

\title{
Mitochondrial Dysfunction and Therapeutic Targets in Auditory Neuropathy
}

\author{
Baoyi Feng, ${ }^{1,2,3}$ Chenxi Jin, ${ }^{1,2,3}$ Zhenzhe Cheng, ${ }^{1,2,3}$ Xingle Zhao, ${ }^{1,2,3}$ Zhuoer Sun, ${ }^{1,2,3}$ \\ Xiaofei Zheng, ${ }^{1,2,3}$ Xiang Li, ${ }^{1,2,3}$ Tingting Dong, ${ }^{4}$ Yong Tao $\mathbb{D}^{1,2,3}$ and Hao Wu $\mathbb{D}^{1,2,3}$ \\ ${ }^{1}$ Department of Otolaryngology-Head and Neck Surgery, Shanghai Ninth People's Hospital, Shanghai Jiaotong University School \\ of Medicine, No. 639, Zhizaoju Road, Shanghai 200011, China \\ ${ }^{2}$ Ear Institute, Shanghai Jiaotong University School of Medicine, No. 115, Jinzun Road, Shanghai 200011, China \\ ${ }^{3}$ Shanghai Key Laboratory of Translation Medicine on Ear and Nose Disease, No. 115, Jinzun Road, Shanghai 200011, China \\ ${ }^{4}$ Biobank of Ninth People's Hospital, Shanghai Jiao Tong University School of Medicine, No. 115, Jinzun Road, \\ Shanghai 200011, China
}

Correspondence should be addressed to Yong Tao; taoyent@foxmail.com and Hao Wu; wuhao622@sina.cn

Received 24 April 2020; Revised 27 May 2020; Accepted 11 July 2020; Published 28 August 2020

Academic Editor: Renjie Chai

Copyright (c) 2020 Baoyi Feng et al. This is an open access article distributed under the Creative Commons Attribution License, which permits unrestricted use, distribution, and reproduction in any medium, provided the original work is properly cited.

\begin{abstract}
Sensorineural hearing loss (SNHL) becomes an inevitable worldwide public health issue, and deafness treatment is urgently imperative; yet their current curative therapy is limited. Auditory neuropathies (AN) were proved to play a substantial role in SNHL recently, and spiral ganglion neuron (SGN) dysfunction is a dominant pathogenesis of AN. Auditory pathway is a high energy consumption system, and SGNs required sufficient mitochondria. Mitochondria are known treatment target of SNHL, but mitochondrion mechanism and pathology in SGNs are not valued. Mitochondrial dysfunction and pharmacological therapy were studied in neurodegeneration, providing new insights in mitochondrion-targeted treatment of AN. In this review, we summarized mitochondrial biological functions related to SGNs and discussed interaction between mitochondrial dysfunction and $\mathrm{AN}$, as well as existing mitochondrion treatment for SNHL. Pharmaceutical exploration to protect mitochondrion dysfunction is a feasible and effective therapeutics for AN.
\end{abstract}

\section{Introduction}

Hearing loss is one of the most crucial public health issues. According to the 70th World Health Assembly (WHA), 360 million people are suffering from auditory dysfunction in the world, accounting for $5 \%$ of the world's population. Besides, more than 1000 million juveniles are risky to hearing disorder [1]. Auditory dysfunction causes speech communication barrier, cognitive disorder, psychological isolation, and inferiority but also brings a heavy burden on family and society. SNHL is the major type of deafness, representing damage in the inner ear or auditory nerves that travel from the ear to the brain [2]. The etiology of deafness is complex, and SGNs draw more and more attention recently [3].

AN or auditory disease was first proposed by Kaga et al. [4] and Starr et al. [5] in 1996, referring to an acquired disorder characteristic of slight hearing impairment with wave I-III absence of auditory brainstem response $(\mathrm{ABR})$ and speech recognition disorder, while distortion product otoacoustic emission (DPOAEs) and cochlear microphonic potential (CMs) did not change. AN may present as a sole clinical phenotype or just be one of the symptoms in systematic diseases like hereditary sensorimotor neuropathies (HSMN) or other demyelinating diseases. Pathology evidence demonstrated auditory nerve damage and loss of inner hair cells (IHCs) and ribbon synapses in AN. AN could be aroused by hereditary defects; for instance, mutation of genes encoding otoferlin or vesicular glutamate transporter 3 was found to induce IHC presynaptic and postsynaptic dysfunctions, respectively. And exogenous damage is another key contributor to be reckoned with, including noise exposure, ototoxic 
drugs, hyperbilirubinemia or thiamin deficiency in infant, or presbycusis [6].

Mitochondrion dysfunction is a major reason for neuropathy. Mitochondria, serving as the engines of eukaryotic cells, participate in cellular energy metabolism, ROS generation, calcium homeostasis, and apoptosis. Mitochondria exhibit special dynamic nature, with feature of pluralistic morphology and great interconnectivity, which determine their function and network structure. Mitochondrion dysfunction is a key reason in aging and neurodegeneration like Alzheimer's disease (AD), amyotrophic lateral sclerosis (ALS), Charcot-Marie-Tooth disease (CMT), and optic atrophy [7]. Additionally, association between mitochondrial biology and optic neuropathies were also detailedly illustrated by pathology and relevant molecular and therapeutic targets. Patients with neuropathy including myoclonic epilepsy with ragged-red fibers (MERRF); mitochondrial encephalomyopathy, lactic acidosis, and stroke-like episodes (MELAS); Charcot-Marie-Tooth disease type 2A (CMT2A); and HSMN caused by mitochondrial dysfunction [8] were also observed suffering from sensorineural hearing loss [9, 10]. The mutation of optic atrophy 1 (OPA1), a key protein related to mitochondrial fusion, was proved to cause syndromic autosomal dominant optic atrophy $(\mathrm{DOA}+)$ with auditory dysfunction [11], which reveals to the potential association between auditory nerves and mitochondria in the development of hearing disorders.

Thus, it is of great significance to explore mitochondrial mechanism of auditory neuropathy and may identify the therapeutic target of auditory neuropathy. In this review, we supply a brief introduction in the mitochondrial structure and function which is correlative to auditory neuropathy and illustrate the potential mechanism between mitochondrial dysfunction and auditory neuropathy. Ultimately, we enumerate the effective therapies targeting mitochondrion dysfunction in AN.

\section{Mitochondrial Genome and Function}

2.1. Mitochondrial Genome. Mitochondrial DNA (mtDNA), which is a mitochondrion-specific genetic system, exists as double-stranded circular molecule with a length of $16569 \mathrm{bp}$ in human. Composed of a heavy strand and a light strand, mtDNA encodes 2 rRNAs, 22 tRNAs, and 13 subunits of the proteins and complexes in respiratory chain including COX I, II, and III and ATP synthase [12], illustrating its crucial role in oxidative phosphorylation (OXPHOS). Plenty of mutations in mtDNA are associated with anomalous OXPHOX. The diversity of mtDNA mutation was observed in neurodegeneration due to the neurons vulnerable to energy supply, especially during aging [13]. The deletion of mtDNA aggravated age-related hearing loss at 12 months of Fischer 344 male rats [14], while D257A and $\mathrm{T} 7511 \mathrm{C}$ mutation in mtDNA accelerated the progression of age-related hearing loss and degeneration of HCs and SGNs $[15,16]$. Moreover, mitochondria are sensitive to ROS since excessive ROS impedes unfolding of protein; therefore, ROS induce mtDNA mutation [17].
MtDNA is of maternal inheritance, and the copy number of mtDNA reaches nearly 1000 in majority of cells, hundreds of times as nuclear DNA genomes. Additionally, mitochondrial biogenesis or heteroplasmy occurs independently in cell division, allowing mutated mtDNA distributed unevenly in subcultured cells without efficient repairment, which was observed in most of the mitochondrial disease [18].

2.2. Mitochondrial Homeostasis. Mitochondrion is an organelle with high interconnection and constant movement, forming cellular networks through a dynamic process. Mitochondrial homeostasis refers to the steady status of the mitochondrial network structure between mitochondrial biogenesis and degradation, including mitochondrial fusion and fission, mitophagy, and trafficking. Disorders of mitochondrial homeostasis have been found in aging and plenty of age-related diseases like neurodegeneration and cardiovascular disease.

Mitochondrial biogenesis is a renewed process of mitochondria by growth and division, associated with protein synthesis, import, and assembly under the guidance of nuclear DNA and mtDNA [19]. Fusion acts on mitochondrial remodelling, modulated by proteolytic processing and PINK1-dependent ubiquitination. Fission allows the extraction of damage segment and quality control of mitochondria, which depends on several critical proteins owning highly conserved dynamic GTPase domain. Mitofusins 1 and 2 (Mfn1 and Mfn2) are located in the outer mitochondrial membrane, and Opal was anchored in the inner mitochondrial membrane. Fusion and fission are also involved in the process of mitophagy with the help of dynamic-related protein 1 (Drp1), a crucial mediator of mitochondrial fission assembled with Fis1 after posttranslational modifications, which could accelerate mitochondrial division $[10,20]$. Apoptosis could be activated by means of regulating proapoptotic factors delivered and expressed in the cytoplasm, such as cyto-c and Bcl-2 [21-24].

Mitophagy is a vital process for mitochondrial quality control that could eliminate impaired mitochondria in time. When mitochondrial membrane potential vanished, PINK1 aggregated on the mitochondrial outer membrane with phosphorylation of Mfn2 and Parkin, inducing ubiquitination of multiple downstream proteins. Finally, impaired mitochondria were separated [10]. Besides, mitochondrial renewal and long-distance energy supply rely on mitochondrial trafficking orthodromic and antidromic. It is essential to neuron that their survival leans more heavily on mitochondrial trafficking than other cells for its high energy consumption and unique cellular morphology. Studies demonstrated fundamental significance to mitochondrial trafficking of motor/adaptor complex composed of kinesin, dynein, Milton, and Miro [25]. Mitochondrial trafficking mechanism in neurodegeneration has been widely studied in $\mathrm{AD}$, Parkinson's disease, Huntington's disease, and amyotrophic lateral sclerosis (ALS) [26].

2.3. Mitochondrial Energetic Metabolism. As a cellular energy organ in eukaryote, mitochondria play vital roles in energy metabolism and ATP production through two essential 
process, the citric acid cycle (TCA) and OXPHOS. The TCA cycle is a critical task in aerobic respiration of eukaryotes as well as an ultimate metabolic step of carbohydrates, fats, and proteins. The close loop initiates with citrate production as acetyl-coenzyme A drifted into TCA cycle and ends as fumarate converted into oxaloacetate, in which electron carriers NADH and FADH2 were manufactured and further participating in electron transfer to electron transport chain (ETC) [27]. The OXPHOS system operates as the launch of ETC. ETC is situated in the inner membrane of mitochondria (IMM), performing functions in conveying electrons through complex I-III, cyto-c, and complex IV successively to convert oxygen to water and driving proton gradient production. Coenzyme Q (CoQ) is the key intermediate electron transporter of this process. With the actuation of proton gradient, ATP is released via ADP phosphorylation through complex V (ATP synthase). Nonetheless, there is still a bit of energy that remained besides the portion consumed by ATP synthesis, as the protons are able to leak across IMM and induce ROS generation to mitochondrial matrix via complexes I and III to a great extent [28]. ROS is an indispensable regulator for normal cellular activities covering intercellular communication as the secondary messenger, proliferation, differentiation, and apoptosis, while excessive accumulation of ROS might lead to oxidative damage, cell death, and diseases like cancer as well as neurodegeneration [29].

Besides, mitochondria also impact apoptosis and regulate calcium flux through mitochondrion-associated ER membranes, which not only act as the second messenger but also are essential to neurotransmitter release like glutamine [30]. As there is a high consumption of energy, normal activities of neuron are bound up with functional mitochondria, including auditory nerves.

\section{Mitochondrial Dysfunction in Auditory Neuropathy}

3.1. Auditory Neuropathy and the Role of SGNs in Auditory Pathway. Neuropathy is a common pathology in SNHL, related to age-related hearing loss and noise-induced hearing loss. Significant SGN degeneration followed by age is observed in apical and basal turns of both human and other mammals' cochlea, while inner or outer hair cells (OHCs) remain existing [31-34]. In Alzheimer's disease (AD), a study found significant loss of SGNs, rather than HC death, which could be found in the cochlea of both 9- and 12month-old 3xTg-AD model mice [35]. Meanwhile, it was demonstrated that swollen cochlear nerve dendrites were seen in the first $24 \mathrm{~h}$ after noise exposure which could lead to temporary threshold shifts (TTS), without HC loss [36]. DPOAE threshold shifts were mild, suggesting that neuropathy and loss of ribbon synapse also contributed to the hearing loss prior to OHC damage. OHCs recovered 2 weeks after exposure, but delayed neurodegeneration was still observed for a long time [37]. In addition to aggravation of $\mathrm{ABR}$ threshold and aberrant compound potential of spiral ganglion, impaired SGNs also conduced to degraded precision of acoustic signal encoding and abnormal speech recognition [6].

Most of SGNs are bipolar cells located in Rosenthal's canal around the modiolus, serving as the primary afferent nerves with innervation of the sensory HCs and cochlear nucleus $[38,39]$. About $95 \%$ of SGNs embedded in myelin formed by satellite glial cells are connected to IHCs, named type I SGNs [40]. The rest of the neurons are type II SGNs and act as postsynaptic sites of OHCs. When action potentials of HCs were initiated by acoustic signal, glutamine, the neurotransmitters were released at ribbon synapses, which was highly specific with precise and speedy information transmission, inducing action potential of SGNs through AMPA receptors [41, 42]. Consequently, SGNs gathered sound signals from dendrites and communicated to an auditory nucleus through axon. The average length of fiber between SGN and HCs in human was nearly $32 \mathrm{~mm}$ [43], which required high energetic consumption and protein synthesis to complete long distance transportation [44]. Imperative requirement of energy support by mitochondria in SGNs indicated the contribution of mitochondrial dysfunction may induce auditory neuropathy (Figure 1).

3.2. Mitochondrial Homeostasis in Auditory Neuropathy. Deregulation of mitochondrial homeostatic mechanism might probably contribute to auditory neuropathy, with dysfunctional mitochondrion biogenesis or impaired dynamics. PGC1- $\alpha$, a key regulator of mitochondrial biogenesis, was also found increased in HCs and auditory cortex, which might improve the sensitivity of age-related hearing loss [45-47]. Additionally, it was found that mutation of tRNA 5-methylaminomethyl-2-thiouridylate methyltransferase (TRMU), the tRNA-modified protein, was related to incidence of SNHL [48, 49]. Dysfunction on mitochondrial protein synthesis plays a fundamental role in SNHL development, when tryptophanyl-tRNA synthetase 2 (Wars2) and mitochondrial ribosomal protein S2 (MRPS2), which are critical to the process, were proved to lead to severe SNHL and SGN loss during mutation $[50,51]$. Mitochondrial protein transport dysfunction also drives the development of SNHL, such as GFER, mitochondrial disulfide relay system protein [52], and DDP [53]. Performing as the critical protein of mitochondrial fission, OPA1 R455H missense mutations were also discovered linking to auditory neuropathy. The absence of $\mathrm{ABR}$, serious speech perception impairment with preserved activity of OHCs, points to the damage of IHCs, ribbon synapse, or auditory nerves [54]. PINK1 is widely expressed in mouse cochlea and able to protect SGNs from cisplatin-induced ototoxicity [55]. Conversely, mitophagy deficiency due to Drp-1 inhibition might give rise to age-related hearing loss with impaired mitochondrial membrane potential HC damage [56].

3.3. Redox Homeostasis and Energetic Metabolism in Auditory Neuropathy. Due to abundant antioxidant enzyme and low transfer potential energy, mitochondria with integrated structure and function can defend against the formation of ROS [57]. ROS homeostasis was associated with 

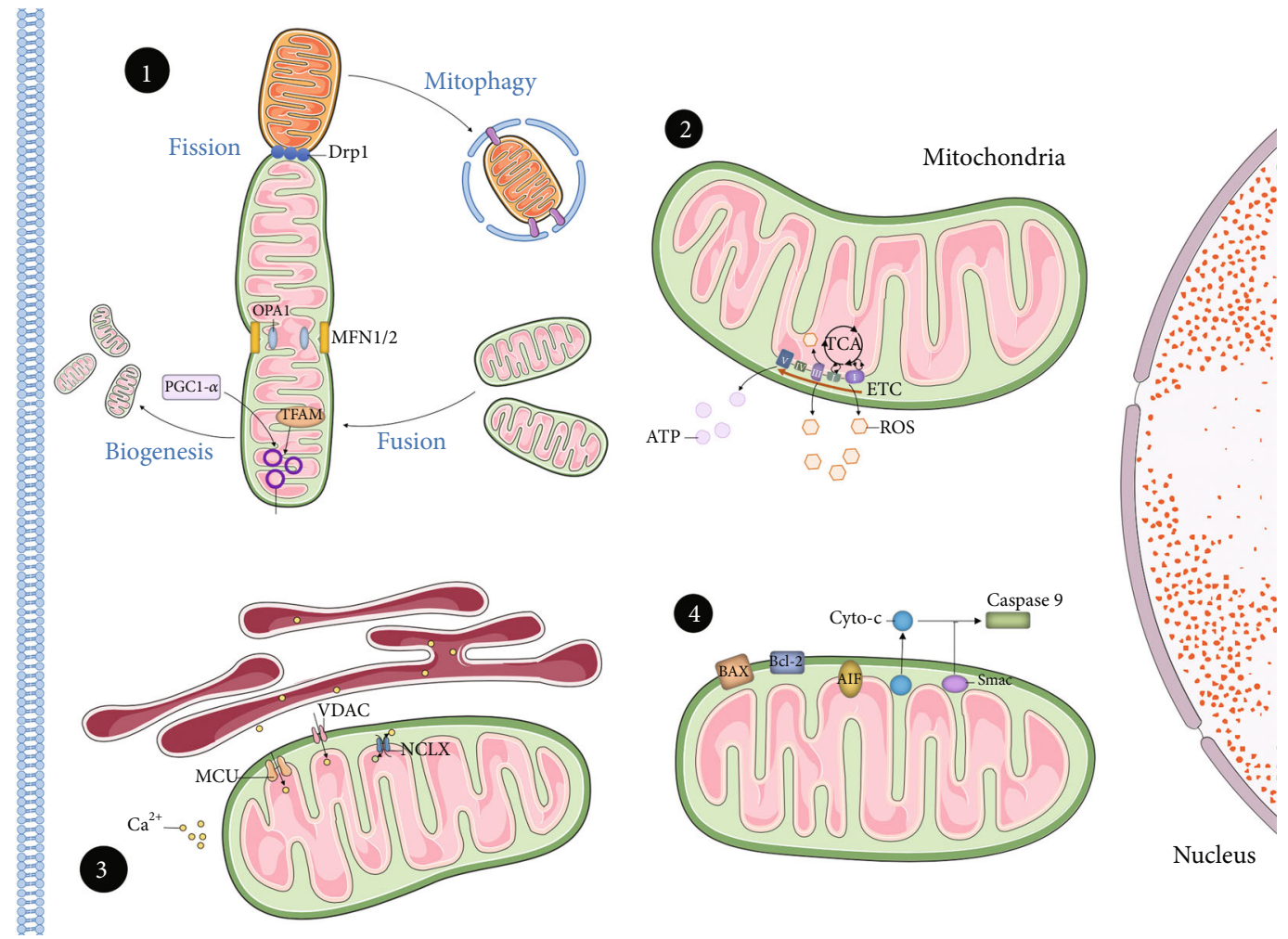

Nucleus

FIGURE 1: Mitochondrial dysfunction mechanism of spiral ganglion neurons in auditory neuropathy. Although mechanisms of mitochondrial dysfunction have not been illustrated distinctly, damages in following targets have been mentioned: (1) mitochondrial homeostasis including biogenesis, dynamics, and mitophagy; (2) redox homeostasis and energetic metabolism; (3) mitochondrial calcium homeostasis; and (4) proapoptotic signal in mitochondria. Drp1: dynamin-related protein 1; MFN1/2: mitofusin 1/2; OPA1: optic atrophy 1; PGC1- $\alpha$ : peroxisome proliferator-activated receptor $\gamma$ coactivator-1 $\alpha$; TFAM: mitochondrial transcription factor A; TCA: tricarboxylic acid; ETC: electron transport chain; MCU: mitochondrial calcium uniporter; VDAC: voltage-dependent anion channel; NCLX: Na+/Ca2+/Li+ exchanger; Bcl-2: B cell lymphoma-2; BAX: Bcl-2 associated protein X; AIF: apoptosis inducing factor.

neurodegeneration and auditory neuropathy [58]. Threeweek-old mice infected with murine congenital cytomegalovirus (MCMV) in neonatal were found to be suffering from hearing loss, and MCMV-infected cultured SGNs in vitro displayed elevated ROS levels and activated NLRP3 inflammasome, which can be suppressed by ROS inhibitor NAC [59]. Additionally, ROS is related to cochlear neuropathy in presbycusis. Evaluated mtDNA oxidative damage and mitochondrial ultrastructural damage in SGNs and auditory cortex were described in aging C57/B6j mice [60]. To mimic human's presbycusis, a senescence-accelerated mouse prone 8 (SAMP8) mouse model was chosen to study the mechanism of ARHL. SGNs of SAMP8 mice own disorganized mitochondria with missing cristae at 12 months, and MDA (a lipid peroxidation) increased and antioxidant enzyme decreased in 1 month, compared to wildtype mice [61]. Disrupted CMP-Neu5Ac hydroxylase (Cmah) is also involved in ARHL. Cmah-null mice showed significant downregulation of ROS gene degradation such as Gpxs and Sod; meanwhile, SGNs lost dramatically. KEGG pathway analysis demonstrated downregulation of mitochondrial molecular transport regulator gene, including Crumbs homolog 1 (Crb1), mitochondrial fission process 1 (Mtfp1), Ras homolog family member T2 (RhoT2), soluble oxidase component (Soc2), and ATP synthase F1 (Atp5f1), indicating mitochondrial dysfunction [62]. The mutation of the protein that can affect ROS production and degradation such as superoxide dismutase (SOD) [63], glutathione Stransferases (GST) [64], mitochondrial uncoupling proteins (UCPs) [65] were found be associated with ARHL.

Now, we have consensus that excessive ROS production aroused cochlear injury in NIHL [66, 67]. Noise exposure induced ROS damage, and raised mitochondrial calcium leads to endoplasmic reticulum (ER) and extracellular fluid, which damage abnormal mitochondrial membrane potential [68-70]. The stria vascularis also contributed to neuropathy: lipid peroxide formation and swollen blood vessels in stria vascularis reduced cochlear blood flow [71, 72], resulting in cochlea ischemia reperfusion and secondary injury by ROS. Noise exposure also caused glutamate excitotoxic neural swelling $[67,73]$. A previous study of excessive ROS production after noise exposure focus on the HCs rather than SGN. Although it was still unknown whether ROS was associated with synapse and SGNs damage in NIHL, SGN was susceptive to hypoxia demonstrated by patients who experienced perinatal and postnatal hypoxia [74].

TCA cycle is a key process for energy-intensive auditory nerves. Isocitrate dehydrogenase 2 (IDH2) is one of the isozymes of IDH and can convert NADP+ to NADPH, involved in TCA cycle. IDH2 dysfunction accelerated apoptosis and 
caused cardiac impairment due to oxidative stress $[75,76]$. Severe oxidative damage and more fragmented nuclear DNA in SGNs were seen in Idh2 $2^{-/-}$mice at 24 months compared to WT, indicating IDH2 deficiency promotes agerelated hearing loss [77]. Calorie restriction protected $\mathrm{HC}$ and SGN degeneration by the promotion of mitochondrial antioxidant defense with sirtuin 3 (Sirt3), which boosted longevity and hearing maintenance [78]. Besides, Sirt3 and Sirt1 help inhibit p53 and restrain apoptosis [79].

3.4. Calcium Homeostasis in Auditory Neuropathy. Calcium ions $\left(\mathrm{Ca}^{2+}\right)$ are secondary messengers in many crucial cellular activities, for instance, cell death and organ development. To maintain proper $\mathrm{Ca}^{2+}$ signaling, a mitochondrion is a vital mediator of calcium in ER, the major intracellular $\mathrm{Ca}^{2+}$ pool. Mitochondrion-associated ER membranes (MAMs), referring to ER-mitochondrion connection, possess calcium transport proteins and channels [80]. MAMs permit fast calcium flux between ER and mitochondrial matrix, which is essential for neural excitation. After being released by ER, calcium ions traverse voltage-dependent anion-selective channel (VDAC) and mitochondrial calcium uniporter (MCU) located in the bilayer of mitochondria and can be extruded to the cytoplasm by sodium calcium exchanger (NCLX) [81]. MCU regulates the activity of enzymes in the TCA cycle [82] and sensitivity of synapses in cochlea to noise exposure. MCU was found to be increased in HCs after noise. Treatment with MCU siRNA or specific MCU inhibitor Ru360 alleviated HCs and ribbon synapse degeneration after noise into $\mathrm{CBA} / \mathrm{J}$ mice. MCU inhibition reduced ABR wave I amplitude damage, suggesting that MCU was correlated to cochlear synaptopathy [83]. Moreover, superfluous calcium uptake results in swollen mitochondria and abnormal mitochondrial membrane potential, inducing mitochondrial apoptotic factors released to the cytoplasm [84].

3.5. Apoptosis in Neuropathy. Mitochondria are of great importance to induce apoptosis under intrinsic and extrinsic stimulations by means of proapoptotic signal like activation of $\mathrm{BH} 3$-only protein or calcium influx and releasing apoptotic protein including cyto-c, caspases, AIF, and Smac $[85,86]$. Abnormal mitochondrial might cause apoptosis in cochlear nerves. Apoptosis-inducing factor (AIF), a flavoprotein and redox enzyme located in mitochondrial intermembrane which can condense chromatin and fracture DNA, was found to be activated by glutamate, which resulted in SGN apoptosis. Calpain was proved to promote mature AIF [87]. Pyridoxine damaged nerve fiber by inducing overload of mitochondrial calcium and activation of apoptosis signal from Bcl-2 family ROS generation and mitochondrial potential transition (MPT) were also aroused after pyridoxine treatment [88]. Although overexpression of bcl-2 might inhibit SGN apoptosis, growth of SGN neurite was suppressed in vitro [89].

\section{Therapy in Auditory Neuropathy}

With the intensive study of mechanism between mitochondrial dysfunction and auditory neuropathy, novel perspec- tives of mitochondrion-targeted therapies were explored. There were several therapies targeting mitochondrial, which will rescue auditory neuropathy fundamentally (Figure 2).

4.1. Antioxidants. Antioxidants were elucidated to protect SNHL by eliminating excessive ROS products, including an intrinsic system such as SODs and GSH and extrinsic system such as inhibitors of calcium, HSP, or salicylate [90].

CoQ10, a common redox in mitochondria and cofactor of respiratory chain, has the capacity of permitting electron and proton transport through ETC and debriding ROS as the antioxidant [91]. Supplementation of water-soluble coenzyme Q10 analog (Qter) alleviated damage of SGNs after noise exposure [92] as well as prevented presbycusis in murine [93].

Methylene blue (MB), distinguished as histological dye, was first applied to clinical practice for the treatment of malaria. Besides, MB could also prevent mitochondria from overproduction of ROS by rerouting electron from NADH to cyto-c and was proved beneficial to neurodegeneration covering NIHL, AD, and PD [94]. Pretreatment with MB diminished ROS and evaluated neurotrophin-3 (NT-3) level, protecting nerve terminals between HCs and SGNs from NIHL [95].

The limitation of the antioxidants was distinct that they could not sweep up ROS in mitochondria precisely and effectively. Recently, studies have shown mitochondrion-targeted antioxidant MitoQ concentrated in solving conventional antioxidant could not aggregate precedingly [96]. MitoQ comprises CoQ10 and lipophilic triphenyl phosphonium (TPP), endowing CoQ10 with the ability to go through a phospholipid bilayer and gather inside mitochondria rapidly, which could stabilized mitochondrial function by enhancing mitochondrial fusion via activation of PGC1- $\alpha$ and upregulation of Mfn2 in the PD model [97]. Besides, other mitochondrion-targeted antioxidants like Mito VitE, and SkQ1 were developed, while the therapeutic effect to auditory neuropathy required verification [98].

4.2. Sirtuin Mediators. Sirtuins are from NAD+-dependent deacylase family which is of great importance to aging and nervous system. SIRT1 participates in the regulation of cellular ROS, synaptic plasticity, and extending lifespan in collaboration with SIRT3, the modulator of mitochondrial metabolism [99]. Sirtuin mediators like resveratrol and NAD + supplement are also popular in antiaging [100], which were also found efficient in NIHL $[101,102]$. Resveratrol, an activator of SIRT1, is a natural antioxidant relevant to mitochondrial biogenesis and modification of mitochondrial function. Mitigatory SGN degeneration and enhancive expression of PINK and Parkin were observed in the mice with long-term replenishment, revealing intensive mitophagy but improved mitochondrial function [100]. Additionally, resveratrol was able to eliminate toxicity protein SGNs from injury caused by noise exposure [101].

NAD, as key coenzyme in several cellular events, took part in the crucial process in mitochondrial metabolism and was associated with axonal degenerations and neurodegeneration. Supplementation of NAD could protect damage 


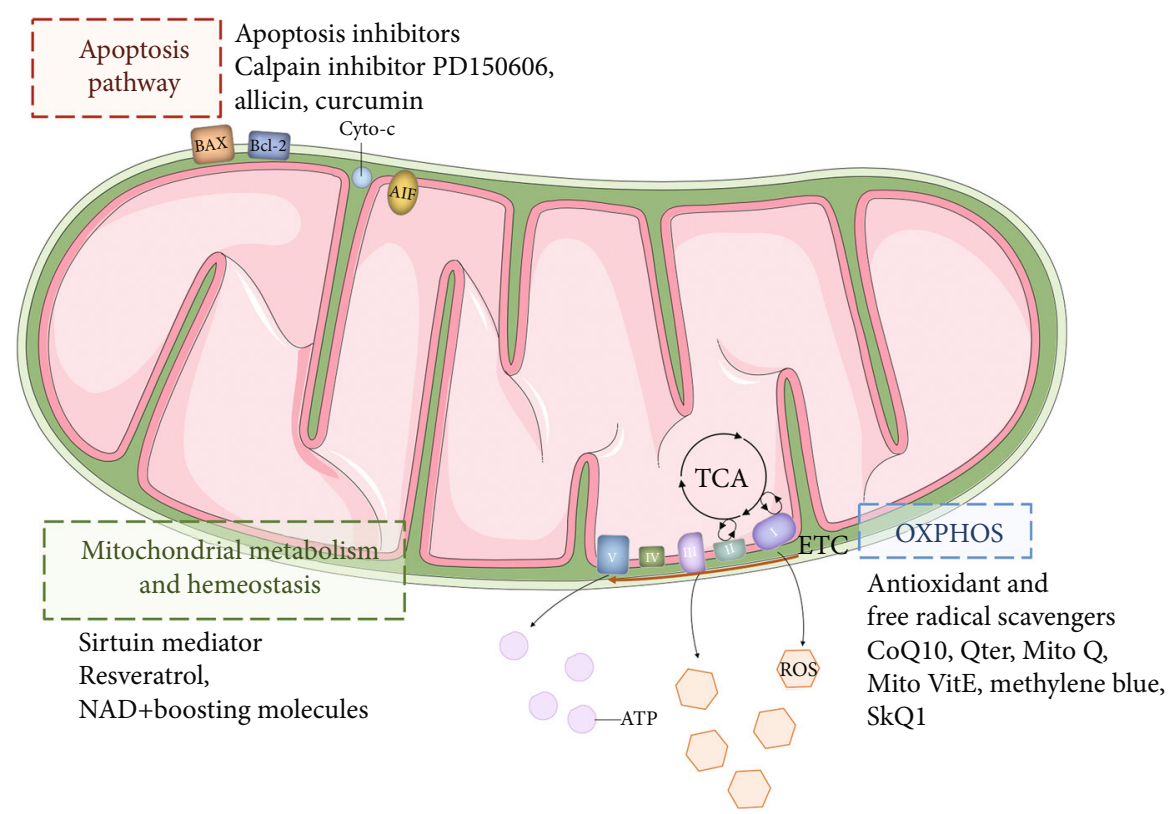

FIgURE 2: Pharmacological targets of mitochondria in auditory neuropathy. Pharmacological therapeutics of mitochondrial dysfunction to rescue auditory neuropathies are still limited. Proven therapeutic strategy targets are comprised of apoptosis inhibition, sirtuin mediators maintaining mitochondrial homeostasis and capability of metabolism, and antioxidants and free radical scavengers that are helpful to alleviate oxidative stress.

neurons and delay neurodegeneration [103]. In hearing loss induced by Mn, NAD was suggested to prevent auditory nerve fibers and SGNs from axonal degeneration and cell apoptosis [102].

4.3. Apoptosis Inhibitors. Due to apoptosis induced by mitochondrial dysfunction, inhibitors of apoptosis targeting mitochondria were developed and found efficient to SNHL. A calpain inhibitor PD150606 could suppress calpain by mediating AIF induced by glutamine and caspase- 12 activation, restraining apoptosis processing and SGNs in vitro [87]. Meanwhile, allicin [104] and curcumin [105] were found to protect SGNs from ototoxic drugs, when paeoniflorin and neurotrophin might exert as protective effect through the PINK1/BAD pathway $[89,106]$.

Others such as gene therapy [107] and stem cell therapy [108] still have been studied. But auditory neuropathy treatment is still limited, requiring more exploration.

\section{Conclusion}

Mitochondrial dysfunction was demonstrated to involve in both hereditary and acquired hearing loss, and the mechanism of ROS damage and mutation of mtDNA in $\mathrm{HC}$ were studied intensively. Mitochondrion function as the energy manufacturer and regulator of apoptosis and calcium homeostasis, which is able to induce SGN damage. The function of mitochondria and the association to neurodegeneration have been excavated, extending perspective on the relationship between mitochondrial dysfunction and auditory neuropathy. Here, we summarized the associ- ation between auditory neuropathy and mitochondrial dysfunction of SGNs, as well as therapeutics targeting mitochondria in AN. Treatments of optic neuropathy including drugs, gene, and stem cell therapies [109] inspired us to explore effective therapeutics for AN.

\section{Conflicts of Interest}

The authors declare that they have no competing interests.

\section{Acknowledgments}

This review was supported by the following foundations: Hao Wu was supported by the Key Project of the National Natural Science Foundation of China (NSFC 81330023), the National Key Technology Research and Development Program of the Ministry of Science and Technology of China (SQ2017YFSF080012), Shanghai Key Laboratory of Translational Medicine on Ear and Nose Diseases (14DZ2260300), Shanghai Municipal Science and Technology Major Project (2018SHZDZX05), and Innovative Research Team of High-Level Local Universities in Shanghai; Yong Tao was supported by the National Natural Science Foundation of China (NSFC 81800900), Shanghai Science and Technology Committee (18411953600, 18ZR1422100, and 18PJ1406900), the Program for Professor of Special Appointment (Eastern Scholar) at Shanghai Institutions of Higher Learning, Shanghai Municipal Health Commission (2018YQ59), Two Hundred Talent of Shanghai Jiaotong University School of Medicine (2019821), and Shanghai Ninth People's Hospital (QC201804). 


\section{References}

[1] World Health Organization, Prevention of deafness and hearing loss, Seventieth World Health Assembly, Geneva, 2017.

[2] Y. Liu, J. Qi, X. Chen et al., "Critical role of spectrin in hearing development and deafness," Science Advances, vol. 5, no. 4, article eaav7803, 2019.

[3] S. Zhang, Y. Zhang, Y. Dong et al., "Knockdown of Foxg1 in supporting cells increases the trans-differentiation of supporting cells into hair cells in the neonatal mouse cochlea," Cellular and Molecular Life Sciences, vol. 77, no. 7, pp. 1401-1419, 2020.

[4] K. Kaga, M. Nakamura, M. Shinogami, T. Tsuzuku, K. Yamada, and M. Shindo, "Auditory nerve disease of both ears revealed by auditory brainstem responses, electrocochleography and otoacoustic emissions," Scandinavian Audiology, vol. 25, no. 4, pp. 233-238, 2009.

[5] A. Starr, T. W. Picton, Y. Sininger, L. J. Hood, and C. I. Berlin, "Auditory neuropathy," Brain, vol. 119, no. 3, pp. 741-753, 1996.

[6] T. Moser and A. Starr, "Auditory neuropathy-neural and synaptic mechanisms," Nature Reviews Neurology, vol. 12, no. 3, pp. 135-149, 2016.

[7] E. A. Schon and S. Przedborski, "Mitochondria: the next (neurode)generation," Neuron, vol. 70, no. 6, pp. 10331053, 2011.

[8] K. W. Chung, S. B. Kim, K. D. Park et al., "Early onset severe and late-onset mild Charcot-Marie-Tooth disease with mitofusin 2 (MFN2) mutations," Brain, vol. 129, no. 8, pp. 21032118, 2006.

[9] S. DiMauro and E. A. Schon, "Mitochondrial disorders in the nervous system," Annual Review of Neuroscience, vol. 31, no. 1, pp. 91-123, 2008.

[10] F. Burté, V. Carelli, P. F. Chinnery, and P. Yu-Wai-Man, "Disturbed mitochondrial dynamics and neurodegenerative disorders," Nature Reviews Neurology, vol. 11, no. 1, pp. 1124, 2015.

[11] A. Starr and G. Rance, “Auditory neuropathy," Handbook of Clinical Neurology, vol. 129, pp. 495-508, 2015.

[12] T. Yasukawa and D. Kang, "An overview of mammalian mitochondrial DNA replication mechanisms," Journal of Biochemistry, vol. 164, no. 3, pp. 183-193, 2018.

[13] N. Nissanka and C. T. Moraes, "Mitochondrial DNA damage and reactive oxygen species in neurodegenerative disease," FEBS Letters, vol. 592, no. 5, pp. 728-742, 2018.

[14] S. Yin, Z. Yu, R. Sockalingam, M. Bance, G. Sun, and J. Wang, "The role of mitochondrial DNA large deletion for the development of presbycusis in Fischer 344 rats," Neurobiology of Disease, vol. 27, no. 3, pp. 370-377, 2007.

[15] B. K. Crawley and E. M. Keithley, "Effects of mitochondrial mutations on hearing and cochlear pathology with age," Hearing Research, vol. 280, no. 1-2, pp. 201-208, 2011.

[16] K. Ishikawa, Y. Tamagawa, K. Takahashi et al., "Temporal bone histopathologic abnormalities associated with mitochondrial mutation T7511C," The Laryngoscope, vol. 116, no. 11, pp. 1982-1986, 2006.

[17] S. Pickles, P. Vigie, and R. J. Youle, "Mitophagy and quality control mechanisms in mitochondrial maintenance," Current Biology, vol. 28, no. 4, pp. R170-R185, 2018.
[18] M. J. Young and W. C. Copeland, "Human mitochondrial DNA replication machinery and disease," Current Opinion in Genetics \& Development, vol. 38, pp. 52-62, 2016.

[19] F. R. Jornayvaz and G. I. Shulman, "Regulation of mitochondrial biogenesis," Essays in Biochemistry, vol. 47, pp. 69-84, 2010.

[20] A. R. Anzell, R. Maizy, K. Przyklenk, and T. H. Sanderson, "Mitochondrial quality control and disease: insights into ischemia-reperfusion injury," Molecular Neurobiology, vol. 55, no. 3, pp. 2547-2564, 2018.

[21] L. L. Xie, F. Shi, Z. Tan, Y. Li, A. M. Bode, and Y. Cao, "Mitochondrial network structure homeostasis and cell death," Cancer Science, vol. 109, no. 12, pp. 3686-3694, 2018.

[22] X. Yu, W. Liu, Z. Fan et al., "c-Myb knockdown increases the neomycin-induced damage to hair-cell-like HEI- OC1 cells in vitro," Scientific Reports, vol. 7, no. 1, article 41094, 2017.

[23] Z. He, L. Guo, Y. Shu et al., "Autophagy protects auditory hair cells against neomycin-induced damage," Autophagy, vol. 13, no. 11, pp. 1884-1904, 2017.

[24] H. Li, Y. Song, Z. He et al., "Meclofenamic acid reduces reactive oxygen species accumulation and apoptosis, inhibits excessive autophagy, and protects hair cell-like HEI-OC1 cells from cisplatin-induced damage," Frontiers in Cellular Neuroscience, vol. 12, p. 139, 2018.

[25] T. L. Schwarz, "Mitochondrial trafficking in neurons," Cold Spring Harbor Perspectives in Biology, vol. 5, no. 6, 2013.

[26] J. Gao, L. Wang, J. Liu, F. Xie, B. Su, and X. Wang, "Abnormalities of mitochondrial dynamics in neurodegenerative diseases," Antioxidants, vol. 6, no. 2, p. 25, 2017.

[27] I. Martinez-Reyes and N. S. Chandel, "Mitochondrial TCA cycle metabolites control physiology and disease," Nature Communications, vol. 11, no. 1, p. 102, 2020.

[28] J. A. Stuart, S. Cadenas, M. B. Jekabsons, D. Roussel, and M. D. Brand, "Mitochondrial proton leak and the uncoupling protein 1 homologues," Biochimica et Biophysica Acta, vol. 1504, no. 1, pp. 144-158, 2001.

[29] L. Milkovic, A. Cipak Gasparovic, M. Cindric, P. A. Mouthuy, and N. Zarkovic, "Short overview of ROS as cell function regulators and their implications in therapy concepts," Cells, vol. 8, no. 8, p. 793, 2019.

[30] J. Nunnari and A. Suomalainen, "Mitochondria: in sickness and in health," Cell, vol. 148, no. 6, pp. 1145-1159, 2012.

[31] E. M. Keithley and M. L. Feldman, "Spiral ganglion cell counts in an age-graded series of rat cochleas," The Journal of Comparative Neurology, vol. 188, no. 3, pp. 429-441, 1979.

[32] E. M. Keithley, "Pathology and mechanisms of cochlear aging," Journal of Neuroscience Research, pp. 1-11, 2019.

[33] C. A. Makary, J. Shin, S. G. Kujawa, M. C. Liberman, and S. N. Merchant, "Age-related primary cochlear neuronal degeneration in human temporal bones," Journal of the Association for Research in Otolaryngology, vol. 12, no. 6, pp. 711-717, 2011.

[34] L. M. Viana, J. T. O'Malley, B. J. Burgess et al., "Cochlear neuropathy in human presbycusis: confocal analysis of hidden hearing loss in post-mortem tissue," Hearing Research, vol. 327, pp. 78-88, 2015.

[35] S. E. Wang and C. H. Wu, "Physiological and histological evaluations of the cochlea between 3xTg-AD mouse model of Alzheimer's diseases and R6/2 mouse model of Huntington's diseases," The Chinese Journal of Physiology, vol. 58, no. 6, pp. 359-366, 2015. 
[36] D. Robertson, "Functional significance of dendritic swelling after loud sounds in the guinea pig cochlea," Hearing Research, vol. 9, no. 3, pp. 263-278, 1983.

[37] S. G. Kujawa and M. C. Liberman, "Adding insult to injury: cochlear nerve degeneration after "temporary" noiseinduced hearing loss," The Journal of Neuroscience, vol. 29, no. 45, pp. 14077-14085, 2009.

[38] Z. Rusznak and G. Szucs, "Spiral ganglion neurones: an overview of morphology, firing behaviour, ionic channels and function," Pflügers Archiv, vol. 457, no. 6, pp. 1303-1325, 2009.

[39] A. M. Berglund and D. K. Ryugo, "Hair cell innervation by spiral ganglion neurons in the mouse," The Journal of Comparative Neurology, vol. 255, no. 4, pp. 560-570, 1987.

[40] W. Liu, M. Boström, A. Kinnefors, F. Linthicum, and H. Rask-Andersen, "Expression of myelin basic protein in the human auditory nerve-an immunohistochemical and comparative study," Auris Nasus Larynx, vol. 39, no. 1, pp. 18-24, 2012.

[41] E. Glowatzki and P. A. Fuchs, "Transmitter release at the hair cell ribbon synapse," Nature Neuroscience, vol. 5, no. 2, pp. 147-154, 2002.

[42] C. Wichmann and T. Moser, "Relating structure and function of inner hair cell ribbon synapses," Cell and Tissue Research, vol. 361, no. 1, pp. 95-114, 2015.

[43] F. Rattay, T. Potrusil, C. Wenger, A. K. Wise, R. Glueckert, and A. Schrott-Fischer, "Impact of morphometry, myelinization and synaptic current strength on spike conduction in human and cat spiral ganglion neurons," PLoS One, vol. 8, no. 11, article e79256, 2013.

[44] Y. Takihara, M. Inatani, K. Eto et al., "In vivo imaging of axonal transport of mitochondria in the diseased and aged mammalian CNS," Proceedings of the National Academy of Sciences of the United States of America, vol. 112, no. 33, pp. 10515-10520, 2015.

[45] S. Hao, L. Wang, K. Zhao, X. Zhu, and F. Ye, "Rs1894720 polymorphism in MIAT increased susceptibility to agerelated hearing loss by modulating the activation of miR29b/SIRT1/PGC-1 $\alpha$ signaling," Journal of Cellular Biochemistry, vol. 120, no. 4, pp. 4975-4986, 2019.

[46] X. Y. Zhao, J. L. Sun, Y. J. Hu et al., "The effect of overexpression of PGC- $1 \alpha$ on the mtDNA4834 common deletion in a rat cochlear marginal cell senescence model," Hearing Research, vol. 296, pp. 13-24, 2013.

[47] Y. Zhong, Y. Hu, W. Peng et al., "Age-related decline of the cytochrome coxidase subunit expression in the auditory cortex of the mimetic aging rat model associated with the common deletion," Hearing Research, vol. 294, no. 1-2, pp. 40$48,2012$.

[48] Z. He, S. Sun, M. Waqas et al., "Reduced TRMU expression increases the sensitivity of hair-cell-like HEI-OC-1 cells to neomycin damage in vitro," Scientific Reports, vol. 6, no. 1, article 29621, 2016

[49] Q. Zhang, L. Zhang, D. Chen et al., "Deletion of Mtu1 (Trmu) in zebrafish revealed the essential role of tRNA modification in mitochondrial biogenesis and hearing function," Nucleic Acids Research, vol. 46, no. 20, pp. 10930-10945, 2018.

[50] T. Gardeitchik, M. Mohamed, B. Ruzzenente et al., "Bi-allelic mutations in the mitochondrial ribosomal protein MRPS2 cause sensorineural hearing loss, hypoglycemia, and multiple
OXPHOS complex deficiencies," American Journal of Human Genetics, vol. 102, no. 4, pp. 685-695, 2018.

[51] T. Agnew, M. Goldsworthy, C. Aguilar et al., "A Wars2 mutant mouse model displays OXPHOS deficiencies and activation of tissue-specific stress response pathways," Cell Reports, vol. 25, no. 12, pp. 3315-3328.e6, 2018.

[52] A. Di Fonzo, D. Ronchi, T. Lodi et al., "The mitochondrial disulfide relay system protein GFER is mutated in autosomalrecessive myopathy with cataract and combined respiratorychain deficiency," American Journal of Human Genetics, vol. 84, no. 5, pp. 594-604, 2009.

[53] F. Bahmad, S. N. Merchant, J. B. Nadol, and L. Tranebjærg, "Otopathology in Mohr-Tranebjaerg syndrome," Laryngoscope, vol. 117, no. 7, pp. 1202-1208, 2007.

[54] R. Santarelli, R. Rossi, P. Scimemi et al., "OPA1-related auditory neuropathy: site of lesion and outcome of cochlear implantation," Brain, vol. 138, no. 3, pp. 563-576, 2015.

[55] Q. Yang, G. Sun, H. Yin et al., "PINK1 protects auditory hair cells and spiral ganglion neurons from cisplatin- induced ototoxicity via inducing autophagy and inhibiting JNK signaling pathway," Free Radical Biology \& Medicine, vol. 120, pp. 342355,2018

[56] H. Lin, H. Xiong, Z. Su et al., "Inhibition of DRP-1dependent mitophagy promotes cochlea hair cell senescence and exacerbates age-related hearing loss," Frontiers in Cellular Neuroscience, vol. 13, 2019.

[57] M. T. Lin and M. F. Beal, "Mitochondrial dysfunction and oxidative stress in neurodegenerative diseases," Nature, vol. 443, no. 7113, pp. 787-795, 2006.

[58] W. Liu, X. Xu, Z. Fan et al., "Wnt signaling activates TP53induced glycolysis and apoptosis regulator and protects against cisplatin-induced spiral ganglion neuron damage in the mouse cochlea," Antioxidants \& Redox Signaling, vol. 30, no. 11, pp. 1389-1410, 2019.

[59] W. Zhuang, C. Wang, X. Shi et al., "MCMV triggers ROS/NLRP3-associated inflammasome activation in the inner ear of mice and cultured spiral ganglion neurons, contributing to sensorineural hearing loss," International Journal of Molecular Medicine, vol. 41, no. 6, pp. 3448-3456, 2018.

[60] Z.-D. du, L. He, C. Tu et al., "Mitochondrial DNA 3,860-bp deletion increases with aging in the auditory nervous system of C57BL/6J mice," ORL-Journal for Oto-Rhino-Laryngology Head and Neck Surgery, vol. 81, no. 2-3, pp. 92-100, 2019.

[61] J. Menardo, Y. Tang, S. Ladrech et al., "Oxidative stress, inflammation, and autophagic stress as the key mechanisms of premature age-related hearing loss in SAMP8 mouse cochlea," Antioxidants \& Redox Signaling, vol. 16, no. 3, pp. 263-274, 2012.

[62] D. N. Kwon, W. J. Park, Y. J. Choi, S. Gurunathan, and J. H. Kim, "Oxidative stress and ROS metabolism via downregulation of sirtuin 3 expression in Cmah-null mice affect hearing loss," Aging, vol. 7, no. 8, pp. 579-594, 2015.

[63] L. S. Nolan, B. A. Cadge, M. Gomez-Dorado, and S. J. Dawson, "A functional and genetic analysis of SOD2 promoter variants and their contribution to age-related hearing loss," Mechanisms of Ageing and Development, vol. 134, no. 7-8, pp. 298-306, 2013.

[64] E. van Eyken, G. van Camp, E. Fransen et al., "Contribution of the N-acetyltransferase 2 polymorphism NAT2*6A to age-related hearing impairment," Journal of Medical Genetics, vol. 44, no. 9, pp. 570-578, 2007. 
[65] S. Sugiura, Y. Uchida, T. Nakashima, F. Ando, and H. Shimokata, "The association between gene polymorphisms in uncoupling proteins and hearing impairment in Japanese elderly," Acta Oto-Laryngologica, vol. 130, no. 4, pp. 487-492, 2009.

[66] E. C. Bottger and J. Schacht, "The mitochondrion: a perpetrator of acquired hearing loss," Hearing Research, vol. 303, pp. 12-19, 2013.

[67] D. Henderson, E. C. Bielefeld, K. C. Harris, and B. H. Hu, "The role of oxidative stress in noise-induced hearing loss," Ear and Hearing, vol. 27, no. 1, pp. 1-19, 2006.

[68] P. H. G. M. Willems, R. Rossignol, C. E. J. Dieteren, M. P. Murphy, and W. J. H. Koopman, "Redox homeostasis and mitochondrial dynamics," Cell Metabolism, vol. 22, no. 2, pp. 207-218, 2015.

[69] C. Batandier, X. Leverve, and E. Fontaine, "Opening of the mitochondrial permeability transition pore induces reactive oxygen species production at the level of the respiratory chain complex I," The Journal of Biological Chemistry, vol. 279, no. 17, pp. 17197-17204, 2004.

[70] A. Kurabi, E. M. Keithley, G. D. Housley, A. F. Ryan, and A. C. Y. Wong, "Cellular mechanisms of noise-induced hearing loss," Hearing Research, vol. 349, pp. 129-137, 2017.

[71] P. R. Thorne, A. L. Nuttall, F. Scheibe, and J. M. Miller, "Sound-induced artifact in cochlear blood flow measurements using the laser Doppler flowmeter," Hearing Research, vol. 31, no. 3, pp. 229-234, 1987.

[72] Y. Wang, K. Hirose, and M. C. Liberman, "Dynamics of noise-induced cellular injury and repair in the mouse cochlea," Journal of the Association for Research in Otolaryngology, vol. 3, no. 3, pp. 248-268, 2002.

[73] J. L. Puel, J. Ruel, C. G. d'Aldin, and R. Pujol, "Excitotoxicity and repair of cochlear synapses after noise-trauma induced hearing loss," Neuroreport, vol. 9, no. 9, pp. 2109-2114, 1998.

[74] Y. Orita, I. Sando, M. Miura, S. I. Haginomori, and B. E. Hirsch, "Cochleosaccular pathology after perinatal and postnatal asphyxia: histopathologic findings," Otology \& Neurotology, vol. 23, no. 1, pp. 34-38, 2002.

[75] Z. J. Reitman and H. Yan, "Isocitrate dehydrogenase 1 and 2 mutations in cancer: alterations at a crossroads of cellular metabolism," JNCI-Journal of the National Cancer Institute, vol. 102, no. 13, pp. 932-941, 2010.

[76] H. J. Ku and J. W. Park, "IDH2 deficiency promotes mitochondrial dysfunction and cardiac hypertrophy in mice," European Journal of Clinical Investigation, vol. 45, pp. 1313, 2015.

[77] K. White, M. J. Kim, C. Han et al., "Loss of IDH2 accelerates age-related hearing loss in male mice," Scientific Reports, vol. 8, no. 1, p. 5039, 2018.

[78] C. Han and S. Someya, "Maintaining good hearing: calorie restriction, Sirt3, and glutathione," Experimental Gerontology, vol. 48, no. 10, pp. 1091-1095, 2013.

[79] L. Bordone and L. Guarente, "Calorie restriction, SIRT1 and metabolism: understanding longevity," Nature Reviews Molecular Cell Biology, vol. 6, no. 4, pp. 298-305, 2005.

[80] S. Marchi, M. Bittremieux, S. Missiroli et al., "Endoplasmic reticulum-mitochondria communication through $\mathrm{Ca}^{2+}$ signaling: the importance of mitochondria-associated membranes (MAMs)," in Organelle Contact Sites. Advances in Experimental Medicine and Biology, vol 997, M. Tagaya and T. Simmen, Eds., pp. 49-67, Springer, Singapore, 2017.
[81] C. Giorgi, S. Marchi, and P. Pinton, "Publisher Correction: The machineries, regulation and cellular functions of mitochondrial calcium," Nature Reviews Molecular Cell Biology, vol. 19, no. 11, pp. 746-746, 2018.

[82] G. S. B. Williams, L. Boyman, and W. J. Lederer, "Mitochondrial calcium and the regulation of metabolism in the heart," Journal of Molecular and Cellular Cardiology, vol. 78, pp. 3545, 2015.

[83] X. Wang, Y. Zhu, H. Long et al., "Mitochondrial calcium transporters mediate sensitivity to noise-induced losses of hair cells and cochlear synapses," Frontiers in Molecular Neuroscience, vol. 11, p. 469, 2019.

[84] C. Giorgi, F. Baldassari, A. Bononi et al., "Mitochondrial Ca ${ }^{2+}$ and apoptosis," Cell Calcium, vol. 52, no. 1, pp. 36-43, 2012.

[85] S. Y. Jeong and D. W. Seol, "The role of mitochondria in apoptosis," BMB Reports, vol. 41, no. 1, pp. 11-22, 2008.

[86] S. Matsuyama and J. C. Reed, "Mitochondria-dependent apoptosis and cellular $\mathrm{pH}$ regulation," Cell Death and Differentiation, vol. 7, no. 12, pp. 1155-1165, 2000.

[87] Z. J. Ding, X. Chen, X. X. Tang et al., "Calpain inhibitor PD150606 attenuates glutamate induced spiral ganglion neuron apoptosis through apoptosis inducing factor pathway in vitro," PLoS One, vol. 10, no. 4, 2015.

[88] C. Park, H. Lim, S. K. Moon, and R. Park, "Pyridoxine preferentially induces auditory neuropathy through mitochondrial dysfunction and endoplasmic reticulum stress-mediated apoptosis," Annals of Otology Rhinology and Laryngology, vol. 128, Supplement 6, pp. 117s-124s, 2019.

[89] J. P. Renton, N. Xu, J. J. Clark, and M. R. Hansen, "Interaction of neurotrophin signaling with Bcl-2 localized to the mitochondria and endoplasmic reticulum on spiral ganglion neuron survival and neurite growth," Journal of Neuroscience Research, vol. 88, no. 10, pp. 2239-2251, 2010.

[90] E. Tavanai and G. Mohammadkhani, "Role of antioxidants in prevention of age-related hearing loss: a review of literature," European Archives of Oto-Rhino-Laryngology, vol. 274, no. 4, pp. 1821-1834, 2017.

[91] H. N. Bhagavan and R. K. Chopra, "Coenzyme Q10: absorption, tissue uptake, metabolism and pharmacokinetics," Free Radical Research, vol. 40, no. 5, pp. 445-453, 2009.

[92] A. R. Fetoni, P. de Bartolo, S. L. M. Eramo et al., "Noiseinduced hearing loss (NIHL) as a target of oxidative stressmediated damage: cochlear and cortical responses after an increase in antioxidant defense," Journal of Neuroscience, vol. 33, no. 9, pp. 4011-4023, 2013.

[93] L. Guastini, R. Mora, M. Dellepiane, V. Santomauro, M. Giorgio, and A. Salami, "Water-soluble coenzyme Q10 formulation in presbycusis: long-term effects," Acta Oto-Laryngologica, vol. 131, no. 5, pp. 512-517, 2010.

[94] D. Tucker, Y. Lu, and Q. Zhang, "From mitochondrial function to neuroprotection-an emerging role for methylene blue," Molecular Neurobiology, vol. 55, no. 6, pp. 51375153, 2018.

[95] J. S. Park, I. Jou, and S. M. Park, "Attenuation of noiseinduced hearing loss using methylene blue," Cell Death \& Disease, vol. 5, no. 4, article e1200, 2014.

[96] Y. R. Kim, J. I. Baek, S. H. Kim et al., “Therapeutic potential of the mitochondria-targeted antioxidant MitoQ in mitochondrial-ROS induced sensorineural hearing loss caused by Idh2 deficiency," Redox Biology, vol. 20, pp. 544555,2019 . 
[97] Y. Xi, D. Feng, K. Tao et al., "MitoQ protects dopaminergic neurons in a 6-OHDA induced PD model by enhancing Mfn2-dependent mitochondrial fusion via activation of PGC-1 $\alpha$," Biochimica et Biophysica Acta (BBA) - Molecular Basis of Disease, vol. 1864, no. 9, pp. 2859-2870, 2018.

[98] C. Fujimoto and T. Yamasoba, "Mitochondria-targeted antioxidants for treatment of hearing loss: a systematic review," Antioxidants, vol. 8, no. 4, p. 109, 2019.

[99] C. K. Singh, G. Chhabra, M. A. Ndiaye, L. M. Garcia-Peterson, N. J. Mack, and N. Ahmad, "The role of sirtuins in antioxidant and redox signaling," Antioxidants \& Redox Signaling, vol. 28, no. 8, pp. 643-661, 2018.

[100] H. Xiong, S. Chen, L. Lai et al., "Modulation of miR34a/SIRT1 signaling protects cochlear hair cells against oxidative stress and delays age-related hearing loss through coordinated regulation of mitophagy and mitochondrial biogenesis," Neurobiology of Aging, vol. 79, pp. 30-42, 2019.

[101] H. Xiong, Y. Ou, Y. Xu et al., "Resveratrol promotes recovery of hearing following intense noise exposure by enhancing cochlear SIRT1 activity," Audiology and Neurotology, vol. 22, no. 3-5, pp. 303-310, 2018.

[102] L. Wang, D. Ding, R. Salvi, and J. A. Roth, "Nicotinamide adenine dinucleotide prevents neuroaxonal degeneration induced by manganese in cochlear organotypic cultures," Neurotoxicology, vol. 40, pp. 65-74, 2014.

[103] L. Rajman, K. Chwalek, and D. A. Sinclair, "Therapeutic potential of NAD-boosting molecules: the in vivo evidence," Cell Metabolism, vol. 27, no. 3, pp. 529-547, 2018.

[104] X. Wu, X. Li, Y. Song et al., “Allicin protects auditory hair cells and spiral ganglion neurons from cisplatin - induced apoptosis," Neuropharmacology, vol. 116, pp. 429-440, 2017.

[105] W. Liu, Z. Fan, Y. Han et al., "Curcumin attenuates peroxynitrite-induced neurotoxicity in spiral ganglion neurons," Neurotoxicology, vol. 32, no. 1, pp. 150-157, 2011.

[106] X. Yu, R. Man, Y. Li et al., "Paeoniflorin protects spiral ganglion neurons from cisplatin-induced ototoxicity: possible relation to PINK1/BAD pathway," Journal of Cellular and Molecular Medicine, vol. 23, no. 8, pp. 5098-5107, 2019.

[107] H. Staecker, W. Liu, B. Malgrange, P. P. Lefebvre, and T. R. van de Water, "Vector-mediated delivery of bcl-2 prevents degeneration of auditory hair cells and neurons after injury," ORL-Journal for Oto-Rhino-Laryngology and Its Related Specialties, vol. 69, no. 1, pp. 43-50, 2006.

[108] Y. H. Hsu, Y. T. Wu, C. Y. Huang et al., "Generation of an induced pluripotent stem cell line from a 39-year-old female patient with severe-to-profound non-syndromic sensorineural hearing loss and a A1555G mutation in the mitochondrial MTRNR1 gene," Stem Cell Research, vol. 25, pp. 245-249, 2017.

[109] M. I. G. Lopez Sanchez, J. G. Crowston, D. A. Mackey, and I. A. Trounce, "Emerging mitochondrial therapeutic targets in optic neuropathies," Pharmacology \& Therapeutics, vol. 165, pp. 132-152, 2016. 